stichting

mathematisch

centrum

$B_{3}$

AFDELING MATHEMATISCHE STATISTIEK

J. OOSTERHOFF \& W.R. VAN ZWET

A NOTE ON CONTIGUITY AND HELLINGER DISTANCE

$2 e$ boerhaavestraat 49 amsterdam 
Printed at the Mathematical Centre, 49, 2 e Boerhaavestraat, Amsterdam.

The Mathematical Centre, founded the 11-th of February 1946, is a nonprofit institurion aiming at the promotion of pure mathematics and its applications. It is sponsored by the Netherlands Government through the Netherlands Organization for the Advancement of Pure Research (Z.W.O), by the Municipality of Amsterdam, by the University of Amsterdam, by the Free University at Amsterdam, and by industries. 


\begin{abstract}
A note on contiguity and Hellinger distance
by
\end{abstract}

J. Oosterhoff \& W.R. van Zwet

ABSTRACT

Two sequences of product probability measures $\left\{\Pi_{i=1}^{n} P_{n i}\right\}$ and $\left\{\Pi_{i=1}^{n} Q_{n i}\right\}$ are considered. Necessary and sufficient conditions in terms of the marginal distributions $P_{n i}$ and $Q_{n i}$ are derived for the contiguity of the sequences. Boundedness for $n \rightarrow \infty$ of the sum of squares of the Hellinger distances of the marginals is one of the conditions. By strengthening these conditions one obtains sufficient and (almost) necessary conditions for the asymptotic normality of the $\log$ likelihood ratio statistic.

KEY WORDS \& PHRASES: asymptotic normality, contiguity, Hellinger distance, log likelihood ratio 
1. INTRODUCTION

For $n=1,2, \ldots$ let $\left(X_{n 1}, A_{n 1}\right), \ldots,\left(X_{n n}, A_{n n}\right)$ be arbitrary measurable spaces. Let $P_{n i}$ and $Q_{n i}$ be probability measures defined on $\left(X_{n i}, A_{n i}\right)$, $i=1, \ldots, n ; n=1,2, \ldots$, and let $P_{n}^{(n)}=\Pi_{i=1}^{n} P_{n i}$ and $Q_{n}^{(n)}=\Pi_{i=1}^{n} Q_{n i}$ $(n=1,2, \ldots)$ denote the product probability measures. For each $i$ and $n$ let $X_{n i}$ be the identity map from $X_{n i}$ onto $X_{n i}$. Then $P_{n i}$ and $Q_{n i}$ represent the two possible distributions of the random element $x_{n i}$ as well as the probability measures of the underlying probability space. Obviously $x_{n 1}, \ldots, x_{n n}$ are independent under both $P_{n}^{(n)}$ and $Q_{n}^{(n)}(n=1,2, \ldots)$.

The sequence $\left\{Q_{n}^{(n)}\right\}$ is said to be contiguous with respect to the sequence $\left\{P_{n}^{(n)}\right\}$ if $\lim _{n \rightarrow \infty} P_{n}^{(n)}\left(A_{n}\right)=0$ implies $\lim _{n \rightarrow \infty} Q_{n}^{(n)}\left(A_{n}\right)=0$ for any sequence of measurable sets $A_{n}$. This one-sided contiguity notion is denoted by $\left\{Q_{n}^{(n)}\right\} \triangleleft\left\{P_{n}^{(n)}\right\}$ (the notation is due to H. WITTING \& G. NÖLLE [7]). The sequences $\left\{P_{n}^{(n)}\right\}$ and $\left\{Q_{n}^{(n)}\right\}$ are said to be contiguous with respect to each other if both $\left\{Q_{n}^{(n)}\right\} \triangleleft\left\{P_{n}^{(n)}\right\}$ and $\left\{P_{n}^{(n)}\right\} \triangleleft\left\{Q_{n}^{(n)}\right\}$. This two-sided contiguity concept we denote by $\left\{P_{n}^{(n)}\right\} \diamond\left\{Q_{n}^{(n)}\right\}^{n}$.

The main purpose of this note is to characterize contiguity of product probability measures in terms of their marginals. To this end we introduce the Hellinger distance $H(P, Q)$ between two probability measures $P$ and $Q$ on the same $\sigma-f i e l d$, defined by

$$
H(P, Q)=\left\{\int\left(p^{\frac{1}{2}}-q^{\frac{1}{2}}\right)^{2} d \mu\right\}^{\frac{1}{2}}=\left\{2-2 \int p^{\frac{1}{2}} q^{\frac{1}{2}} d \mu\right\}^{\frac{1}{2}},
$$

where $\mathrm{p}=\mathrm{dP} / \mathrm{d} \mu, \mathrm{q}=\mathrm{dQ} / \mathrm{d} \mu$ and $\mu$ is any $\sigma$-finite measure dominating $\mathrm{P}+\mathrm{Q}$.

This metric is independent of the choice of $\mu$ and satisfies $0 \leq H(P, Q) \leq 2^{\frac{1}{2}}$. Defining the total variation distance of $P$ and $Q$ by

$$
\|P-Q\|=\sup |P(A)-Q(A)| \text {, }
$$

where the supremum is taken over all measurable sets $A$, we have the following inequalities (LECAM [4])

$$
\frac{1}{2} H^{2}(P, Q) \leq\|P-Q\| \leq H(P, Q)
$$


The Hellinger distances of the product measures and of their marginals are connected by the relationship

$$
H^{2}\left(P_{n}^{(n)}, Q_{n}^{(n)}\right)=2-2 \Pi_{i=1}^{n}\left\{1-\frac{1}{2} H^{2}\left(P_{n i}, Q_{n i}\right)\right\} .
$$

For further reference we first mention two easy results, viz.

$$
\sum_{i=1}^{n} H^{2}\left(P_{n i}, Q_{n i}\right)=o(1) \quad \text { for } n \rightarrow \infty \Rightarrow\left\{P_{n}^{(n)}\right\} \triangleleft\left\{Q_{n}^{(n)}\right\} \text {, }
$$

and

$$
\left\{Q_{n}^{(n)}\right\} \triangleleft\left\{P_{n}^{(n)}\right\} \Rightarrow \sum_{i=1}^{n} H^{2}\left(P_{n i}, Q_{n i}\right)=O(1) \text { for } n \rightarrow \infty
$$

The proof of (1.5) is an immediate consequence of the string of implications $\sum_{i=1}^{\mathrm{n}} \mathrm{H}^{2}\left(\mathrm{P}_{\mathrm{ni}}, \mathrm{Q}_{\mathrm{ni}}\right)=o(1) \Rightarrow \sum_{\mathrm{i}=1}^{\mathrm{n}} \log \left\{1-\frac{1}{2} \mathrm{H}^{2}\left(\mathrm{P}_{\mathrm{ni}}, \mathrm{Q}_{\mathrm{ni}}\right)\right\}=o(1) \Rightarrow$ $\Rightarrow H^{2}\left(P_{n}^{(n)}, Q_{n}^{(n)}\right)=o(1) \Rightarrow\left\|P_{n}^{(n)}-Q_{n}^{(n)}\right\|=O(1) \Rightarrow\left\{P_{n}^{(n)}\right\} \triangleleft\left\{Q_{n}^{(n)}\right\}$. To prove (1.6) suppose that $\limsup _{n \rightarrow \infty} H\left(P_{n}^{(n)}, Q_{n}^{(n)}\right)=2^{\frac{1}{2}}$. Then by (1.3) $\limsup _{n \rightarrow \infty}\left\|P_{n}^{(n)}-Q_{n}^{(n)}\right\|=1$ in contradiction to $\left\{Q_{n}^{(n)}\right\} \triangleleft\left\{P_{n}^{(n)}\right\}$. Thus $\limsup _{n \rightarrow \infty} H^{2}\left(P_{n}^{(n)}, Q_{n}^{(n)}\right)<2$, therefore $\liminf { }_{n \rightarrow \infty} \Pi_{i=1}^{n}\left\{1-\frac{1}{2} H^{2}\left(P_{n i}, Q_{n i}\right)\right\}>0$ and hence limsup $_{n \rightarrow \infty} \sum_{i=1}^{n} H^{2}\left(P_{n i}, Q_{n i}\right)<\infty$ and the proof is complete.

It can be shown by counterexamples that in (1.5) the condition cannot be weakened to $\sum_{i=1}^{n} H^{2}\left(P_{n i}, Q_{n i}\right)=O(1)$, and that in (1.6) the conclusion cannot be strengthened to $\sum_{i=1}^{n} H^{2}\left(P_{n i}, Q_{n i}\right)=o(1)$, for $n \rightarrow \infty$. Hence there remains a gap between the sufficient condition and the necessary condition for contiguity in (1.5) and (1.6) respectively. In section 2 we obtain conditions which are both sufficient and necessary for contiguity of the product measures by adding another condition to $\sum_{i=1}^{n} H^{2}\left(P_{n i}, Q_{n i}\right)=O(1)$.

In many applications asymptotic normality of the log likelihood ratio statistic $\Lambda_{\mathrm{n}}$ (see (3.1)) plays an important part. Since $L\left(\Lambda_{n} \mid P_{n}^{(n)}\right) \rightarrow_{w} N\left(-\frac{1}{2} \sigma^{2} ; \sigma^{2}\right)$ implies $\left\{P_{n}^{(n)}\right\} \diamond\left\{Q_{n}^{(n)}\right\}$ (cf. HÁJEK \& ŠIDÁK [1], LECAM [2], [3], [4], ROUSSAS [6]), we have to impose stronger conditions on the marginals $P_{n i}$ and $Q_{n i}$ to ensure the asymptotic normality of $\Lambda_{n}$. Some sufficient (and almost necessary) conditions for the asymptotic normality of $\Lambda_{n}$, which are clearly stronger than those in section 2, are given in section 3 . These conditions are closely related to some earlier results of LECAM [3], [4]. 


\section{CONTIGUITY OF PRODUCT MEASURES}

We begin by noting the following useful implication:

$$
\begin{aligned}
& \left\{Q_{n}^{(n)}\right\} \triangleleft\left\{P_{n}^{(n)}\right\} \Rightarrow \\
& \Rightarrow\left[\lim _{n \rightarrow \infty} \sum_{i=1}^{n} P_{n i}\left(A_{n i}\right)=0 \Rightarrow \lim _{n \rightarrow \infty} \sum_{i=1}^{n} Q_{n i}\left(A_{n i}\right)=0\right]
\end{aligned}
$$

for any collection of measurable sets $A_{\text {ni }}$. For suppose $\lim _{n \rightarrow \infty} \sum_{i=1}^{n} P_{n i}\left(A_{n i}\right)=0$. Then $\lim _{n \rightarrow \infty} P_{n}^{(n)}\left(U_{i=1}^{n} A_{n i}\right)=0$, hence by contiguity $\lim _{n \rightarrow \infty} Q_{n}^{(n)}\left(U_{i=1}^{n} A_{n i}\right)=1-\lim _{n \rightarrow \infty} \Pi_{i=1}^{n}\left(1-Q_{n i}\left(A_{n i}\right)\right)=0$ and therefore $\lim _{n \rightarrow \infty} \sum_{i=1}^{n} Q_{n i}\left(A_{n i}\right)=0$.

Now let $\mu_{n i}$ be a $\sigma$-finite measure on $\left(X_{n i}, A_{n i}\right)$ dominating $P_{n i}+Q_{n i}$ and write $\mathrm{p}_{n i}=\mathrm{dP}_{n i} / \mathrm{d} \mu_{n i}$ and $\mathrm{q}_{n i}=\mathrm{dQ}_{n i} / \mathrm{d} \mu_{n i}(\mathrm{i}=1, \ldots, \mathrm{n} ; \mathrm{n}=1,2, \ldots)$. The main result of this section is

THEOREM $1 \cdot\left\{Q_{\mathrm{n}}^{(\mathrm{n})}\right\} \triangleleft\left\{\mathrm{P}_{\mathrm{n}}^{(\mathrm{n})}\right\}$ iff

$$
\limsup _{n \rightarrow \infty} \sum_{i=1}^{n} H^{2}\left(P_{n i}, Q_{n i}\right)<\infty
$$

and

$$
\lim _{n \rightarrow \infty} \sum_{i=1}^{n} Q_{n i}\left(q_{n i}\left(x_{n i}\right) / p_{n i}\left(x_{n i}\right) \geq c_{n}\right)=0 \text { whenever } c_{n} \rightarrow \infty \text {. }
$$

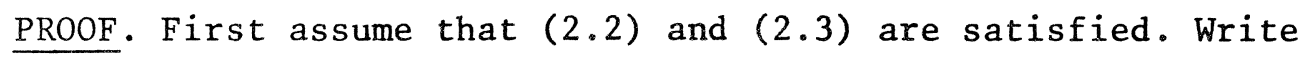

$$
L_{n i}=q_{n i}\left(x_{n i}\right) / p_{n i}\left(x_{n i}\right), \quad i=1, \ldots, n ; n=1,2, \ldots,
$$

and consider $\pi_{i=1}^{n} L_{n i}$. It is easily shown (cf. LECAM [4], ROUSSAS [6]) that $\left\{Q_{n}^{(n)}\right\} \triangleleft\left\{P_{n}^{(n)}\right\}$ is equivalent to tightness of the sequence of distributions $\left\{L\left(\pi_{i=1}^{n} L_{n i} \mid Q_{n}^{(n)}\right) ; n=1,2, \ldots\right\}$. The tightness of this set of distributions can also be expressed in the more convenient form

$$
\lim _{n \rightarrow \infty} Q_{n}^{(n)}\left(\Pi_{i=1}^{n} L_{n i} \geq k_{n}\right)=0 \text { whenever } k_{n} \rightarrow \infty \text {. }
$$


Hence we have to prove $(2.4)$. Let $0<k_{n} \rightarrow \infty$. Let $0<c_{n} \rightarrow \infty$ be real numbers to be chosen in the sequel. If ${ }_{A}$ denotes the indicator function of the set $A$, we have by (2.3) and Markov's inequality for $n \rightarrow \infty$

$$
\begin{aligned}
& Q_{n}^{(n)}\left(\Pi_{i=1}^{n} L_{n i} \geq k_{n}\right) \leq \\
& \leq Q_{n}^{(n)}\left(\Pi_{i=1}^{n} L_{n i} \geq k_{n} \wedge L_{n i}<c_{n} \text { for } i=1, \ldots, n\right)+Q_{n}^{(n)}\left(U_{i=1}^{n}\left\{L_{n i} \geq c_{n}\right\}\right) \leq \\
& \leq Q_{n}^{(n)}\left(\Pi_{i=1}^{n} L_{n i}^{\frac{1}{2}} 1\left(0, c_{n}\right)\left(L_{n i}\right) \geq k_{n}^{\frac{1}{2}}\right)+\sum_{i=1}^{n} Q_{n i}\left(L_{n i} \geq c_{n}\right) \leq \\
& \leq k_{n}^{-\frac{1}{2}} \Pi_{i=1}^{n} \int_{q_{n i}<c_{n} p_{n i}} q_{n i}^{\frac{3}{2}} p_{n i}^{-\frac{1}{2}} d \mu_{n i}+O(1) .
\end{aligned}
$$

Since for a11 $c_{n} \geq 1$

$$
\begin{aligned}
& \int_{q_{n i}<c_{n} p_{n i}} q_{n i}^{\frac{3}{2}} p_{n i}^{-\frac{1}{2}} d \mu_{n i} \leq \\
& \leq \int_{q_{n i}<c} p_{n i} q_{n i} d \mu_{n i}+\int_{q_{n i}<c} p_{n i} q_{n i} p_{n i}^{-\frac{1}{2}}\left(q_{n i}^{\frac{1}{2}}-p_{n i}^{\frac{1}{2}}\right) d \mu_{n i} \leq \\
& \leq 1+\int_{q_{n i}<c} p_{n i} q_{n i}^{\frac{1}{2}} p_{n i}^{-\frac{1}{2}}\left(q_{n i}^{\frac{1}{2}}-p_{n i}^{\frac{1}{2}}\right)^{2} d_{n i}+\int_{q_{n i}<c_{n} p_{n i}} q_{n i}^{\frac{1}{2}}\left(q_{n i}^{\frac{1}{2}}-p_{n i}^{\frac{1}{2}}\right) d \mu_{n i} \leq \\
& \leq 1+c_{n}^{\frac{1}{2}} \int\left(q_{n i}^{\frac{1}{2}}-p_{n i}^{\frac{1}{2}}\right)^{2} d \mu_{n i}+1-\int q_{n i}^{\frac{1}{2}} p_{n i}^{\frac{1}{2}} d \mu_{n i}-\int_{q_{n i} \geq c_{n} p_{n i}} q_{n i}^{\frac{1}{2}}\left(q_{n i}^{\frac{1}{2}}-p_{n i}^{\frac{1}{2}}\right) d \mu_{n i} \leq \\
& \leq 1+\left(c_{n}^{\frac{1}{2}}+\frac{1}{2}\right) H^{2}\left(P_{n i}, Q_{n i}\right),
\end{aligned}
$$

it follows that

$$
\begin{aligned}
& \limsup _{n \rightarrow \infty} Q_{n}^{(n)}\left(\Pi_{i=1}^{n} L_{n i} \geq k_{n}\right) \leq \\
& \leq \limsup _{n \rightarrow \infty} k_{n}^{-\frac{1}{2}} \Pi_{i=1}^{n}\left\{1+\left(c_{n}^{\frac{1}{2}}+\frac{1}{2}\right) H^{2}\left(P_{n i}, Q_{n i}\right)\right\} \leq \\
& \leq \limsup _{n \rightarrow \infty} k_{n}^{-\frac{1}{2}} \exp \left\{\left(c_{n}^{\frac{1}{2}}+\frac{1}{2}\right) \sum_{i=1}^{n} H^{2}\left(P_{n i}, Q_{n i}\right)\right\} .
\end{aligned}
$$


Choosing $c_{n}$ in such a way that $c_{n}=o\left(\left(\log k_{n}\right)^{2}\right)$ for $n \rightarrow \infty,(2.2)$ implies $Q_{n}^{(n)}\left(\pi_{i=1}^{n} L_{n i} \geq k_{n}\right)=O(1)$ for $n \rightarrow \infty$ and $(2.4)$ is established.

Conversely, suppose that $\left\{Q_{n}^{(n)}\right\} \triangleleft\left\{P_{n}^{(n)}\right\}$. Since (1.6) implies that $(2.2)$ is satisfied, it remains to prove (2.3). Let $0<c_{n} \rightarrow \infty$ and consider the inequality, valid for $c_{n} \geq 4$,

$$
\begin{aligned}
& \sum_{i=1}^{n} \int_{q_{n i} \geq c_{n} p_{n i}} p_{n i} d \mu_{n i} \leq c_{n}^{-\frac{1}{2}} \sum_{i=1}^{n} \int_{q_{n i} \geq c_{n} p_{n i}} p_{n i}^{\frac{1}{2}} q_{n i}^{\frac{1}{2}} d \mu_{n i}= \\
& =c_{n}^{-\frac{1}{2}}\left\{\sum_{i=1}^{n} \int_{q_{n i} \geq c_{n} p_{n i}} p_{n i}^{\frac{1}{2}}\left(q_{n i}^{\frac{1}{2}}-p_{n i}^{\frac{1}{2}}\right) d \mu_{n i}+\sum_{i=1}^{n} \int_{q_{n i} \geq c_{n} p_{n i}} p_{n i} d \mu_{n i}\right\} \leq \\
& \leq c_{n}^{-\frac{1}{2}}\left\{\sum_{i=1}^{n} \int_{q_{n i} \geq c_{n} p_{n i}}\left(q_{n i}^{\frac{1}{2}}-p_{n i}^{\frac{1}{2}}\right)^{2} d \mu_{n i}+\sum_{i=1}^{n} \int_{q_{n i} \geq c_{n} p_{n i}} p_{n i} d \mu_{n i}\right\} \leq
\end{aligned}
$$

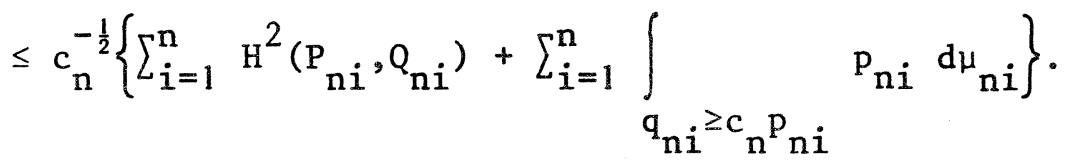

Since by $(2.2) c_{n}^{-\frac{1}{2}} \sum_{i=1}^{n} H^{2}\left(P_{n i}, Q_{n i}\right) \rightarrow 0$ for $n \rightarrow \infty$, it follows that $\lim _{n \rightarrow \infty} \sum_{i=1}^{n} P_{n}\left(L_{n i} \geq c_{n}\right)=0$. Hence (2.1) implies that

$\lim _{n \rightarrow \infty} \sum_{i=1}^{n} Q_{n i}\left(L_{n i} \geq c_{n}\right)=0$ and the proof of the theorem is complete.

COROLLARY 1. $\left\{\mathrm{P}_{\mathrm{n}}^{(\mathrm{n})}\right\} \diamond\left\{\mathrm{Q}_{\mathrm{n}}^{(\mathrm{n})}\right\}$ iff $(2.2)$ and $(2.3)$ are satisfied and

$$
\lim _{n \rightarrow \infty} \sum_{i=1}^{n} P_{n i}\left(p_{n i}\left(x_{n i}\right) / q_{n i}\left(x_{n i}\right) \geq c_{n}\right)=0 \text { whenever } c_{n} \rightarrow \infty \text {. }
$$

In connection with contiguity Hellinger distance seems to be a more appropriate metric than total variation distance. Note that from (1.3) and (1.6) we immediately obtain the implication

$$
\left\{Q_{n}^{(n)}\right\} \triangleleft\left\{P_{n}^{(n)}\right\} \Rightarrow \sum_{i=1}^{n}\left\|P_{n i}-Q_{n i}\right\|^{2}=O(1) \text { for } n \rightarrow \infty,
$$

where again the order term cannot be strenghtened to $O(1)$. However, $\sum_{i=1}^{n}\left\|P_{n i}-Q_{n i}\right\|^{2}=O(1)$ is too weak a condition to replace (2.2) in theo- 
rem 1. On the other hand we cannot strengthen this condition to $\sum_{i=1}^{n}\left\|P_{n i}-Q_{n i}\right\|^{r}=O(1)$ for some $r<2$, since $\left\{Q_{n}^{(n)}\right\} \triangleleft\left\{P_{n}^{(n)}\right\}$ does not necessarily imply $\sum_{i=1}^{n}\left\|P_{n i}-Q_{n i}\right\|^{r}=O(1)$ for any positive $r<2$. The following example serves to illustrate these points.

EXAMPLE. Let $\mu_{n i}$ denote Lebesgue measure on $(0,1)$, let $p_{n i}=1(0,1)$ and let $\mathrm{q}_{n i}=\left(1+n^{-\frac{1}{2}}\right) 1_{\left(0,1-n^{-\frac{1}{2}}\right)}+n^{-\frac{1}{2}} 1_{\left[1-n^{-\frac{1}{2}}, 1\right)}, i=1, \ldots, n ; n=1,2, \ldots$. Then $\sum_{i=1}^{n}\left\|P_{n i}-Q_{n i}\right\|^{2}=\left(1-n^{-\frac{1}{2}}\right)^{2} \leq 1$ and $(2.3)$ is trivially satisfied since $\mathrm{q}_{\mathrm{ni}} / \mathrm{p}_{\mathrm{ni}}$ is uniformly bounded. But $\left\{\mathrm{Q}_{\mathrm{n}}^{(\mathrm{n})}\right\} \triangleleft\left\{\mathrm{P}_{\mathrm{n}}^{(\mathrm{n})}\right\}$ does not hold because $\sum_{i=1}^{n} H^{2}\left(P_{n i}, Q_{n i}\right)=2 n\left\{1-\int q_{n i}^{\frac{1}{2}} d \mu_{n i}\right\}=2 n\left\{1-\left(1+n^{-\frac{1}{2}}\right)^{\frac{1}{2}}\left(1-n^{-\frac{1}{2}}\right)-n^{-\frac{3}{4}}\right\}=$ $=\mathrm{n}^{\frac{1}{2}}(1+O(1))$ for $\mathrm{n} \rightarrow \infty$.

Taking $q_{n i}=\left(1+n^{-\frac{1}{2}}\right) 1_{\left(0, \frac{1}{2}\right)}+\left(1-n^{-\frac{1}{2}}\right) 1_{\left[\frac{1}{2}, 1\right)}$ for all $i$ and $n$, we have $\left\{Q_{n}^{(n)}\right\} \triangleleft\left\{P_{n}^{(n)}\right\}$ since $(2.3)$ is satisfied and $\sum_{i=1}^{n} H^{2}\left(P_{n i}, Q_{n i}\right)=$ $=2 n\left\{1-\frac{1}{2}\left(1+n^{-\frac{1}{2}}\right)^{\frac{1}{2}}-\frac{1}{2}\left(1-n^{-\frac{1}{2}}\right)^{\frac{1}{2}}\right\}=\frac{1}{4}+o(1)$ for $n \rightarrow \infty$. However, in this case $\sum_{i=1}^{n}\left\|P_{n i}-Q_{n i}\right\|^{r}=n\left(\frac{1}{2} n^{-\frac{1}{2}}\right)^{r} \rightarrow \infty$ for $n \rightarrow \infty$ if $r<2$.

3. ASYMPTOTIC NORMALITY OF $\Lambda_{\mathrm{n}}$

Define

$$
\Lambda_{n}=\sum_{i=1}^{n} \log \left\{q_{n i}\left(x_{n i}\right) / p_{n i}\left(x_{n i}\right)\right\}, \quad n=1,2, \ldots .
$$

Note that, with probability one, $\Lambda_{n}$ is well-defined under $P_{n}^{(n)}$, although $\Lambda_{n}$ may assume the value $-\infty$ with positive probability under $P_{n}(n)$.

In our search for necessary and sufficient conditions for the weak convergence $L\left(\Lambda_{n} \mid P_{n}{ }^{(n)}\right) \rightarrow_{w} N\left(-\frac{1}{2} \sigma^{2} ; \sigma^{2}\right)$ in terms of the marginal distributions of the $x_{n i}$ we shall confine ourselves to the case where the summands in (3.1) satisfy the traditional u.a.n. condition (cf. LOĖVE [5]).

THEOREM 2. For any $\sigma \geq 0$

$$
L\left(\Lambda_{n} \mid P_{n}^{(n)}\right) \rightarrow_{w} N\left(-\frac{1}{2} \sigma^{2} ; \sigma^{2}\right)
$$

and 


$$
\lim _{n \rightarrow \infty} \max _{1 \leq i \leq n} P_{n i}\left(\left|\log \left\{q_{n i}\left(x_{n i}\right) / p_{n i}\left(x_{n i}\right)\right\}\right| \geq \varepsilon\right)=0
$$

for every $\varepsilon>0$ iff for every $\varepsilon>0$

$$
\lim _{n \rightarrow \infty} \sum_{i=1}^{n} H^{2}\left(P_{n i}, Q_{n i}\right)=\frac{1}{4} \sigma^{2},
$$

$$
\lim _{n \rightarrow \infty} \sum_{i=1}^{n} Q_{n i}\left(q_{n i}\left(x_{n i}\right) / p_{n i}\left(x_{n i}\right) \geq 1+\varepsilon\right)=0,
$$

$$
\lim _{n \rightarrow \infty} \sum_{i=1}^{n} P_{n i}\left(p_{n i}\left(x_{n i}\right) / q_{n i}\left(x_{n i}\right) \geq 1+\varepsilon\right)=0
$$

or equivalently, iff (3.4) holds and for every $\varepsilon>0$

$$
\lim _{n \rightarrow \infty} \sum_{i=1}^{n} \int_{\left|q_{n i}-p_{n i}\right| \geq \varepsilon p_{n i}}\left(q_{n i}^{\frac{1}{2}}-p_{n i}^{\frac{1}{2}}\right)^{2} d \mu_{n i}=0 .
$$

PROOF. To simplify the notation we write $r_{n i}=q_{n i} / p_{n i}$. We first show that (3.5) and (3.6) are equivalent to (3.7). From

$$
\begin{aligned}
& \sum_{i=1}^{n} \int_{\left|q_{n i}-p_{n i}\right| \geq \varepsilon p_{n i}}\left(q_{n i}^{\frac{1}{2}}-p_{n i}^{\frac{1}{2}}\right)^{2} d \mu_{n i}= \\
& =\sum_{i=1}^{n}\left\{\int_{r_{n i} \geq 1+\varepsilon} q_{n i}\left(1-r_{n i}^{-\frac{1}{2}}\right)^{2} d \mu_{n i}+\int_{r_{n i} \leq 1-\varepsilon} p_{n i}\left(1-r_{n i}^{\frac{1}{2}}\right)^{2} d \mu_{n i}\right\}
\end{aligned}
$$

we obtain the double inequality

$$
\begin{aligned}
& \left\{1-(1+\varepsilon)^{-\frac{1}{2}}\right\}^{2} \sum_{i=1}^{n} Q_{n i}\left(r_{n i}\left(x_{n i}\right) \geq 1+\varepsilon\right)+ \\
& +\left\{1-(1-\varepsilon)^{\frac{1}{2}}\right\}^{2} \sum_{i=1}^{n} P_{n i}\left(r_{n i}^{-1}\left(x_{n i}\right) \geq(1-\varepsilon)^{-1}\right) \leq \\
& \leq \sum_{i=1}^{n} \int_{\left|q_{n i}-p_{n i}\right| \geq \varepsilon p_{n i}}\left(q_{n i}^{\frac{1}{2}}-p_{n i}^{\frac{1}{2}}\right)^{2} d_{n i} \leq \\
& \leq \sum_{i=1}^{n} Q_{n i}\left(r_{n i}\left(x_{n i}\right) \geq 1+\varepsilon\right)+\sum_{i=1}^{n} P_{n i}\left(r_{n i}^{-1}\left(x_{n i}\right) \geq(1-\varepsilon)^{-1}\right)
\end{aligned}
$$


and the equivalence of $(3.5)$ and $(3.6)$ to $(3.7)$ is immediate.

Next we note that both $(3.2),(3.3)$ and $(3.4),(3.5),(3.6)$ imply $\left\{P_{n}^{(n)}\right\} \diamond\left\{Q_{n}^{(n)}\right\}$ (cf. corollary 1 ).

The remainder of the proof relies on the normal convergence theorem

(cf. LOĖVE [5]). According to an equivalent form of this theorem (3.2) and

(3.3) are equivalent to

$$
\lim _{\delta \downarrow 0} \lim _{n \rightarrow \infty} \sum_{i=1}^{n}\left\{\int_{\left|\log r_{n i}\right| \leq \delta}\left(\log r_{n i}\right)^{2} d P_{n i}-\left(\int_{\left|\log r_{n i}\right| \leq \delta}\left(\log r_{n i}\right) d P_{n i}\right)^{2}\right\}=\sigma^{2} .
$$

By the contiguity of $\left\{P_{n}^{(n)}\right\}$ and $\left\{Q_{n}^{(n)}\right\}$ and (2.1) the condition (3.8) is equivalent to (3.5) and (3.6) and hence to (3.7). Henceforth we assume (3.7), (3.8) and $\left\{\mathrm{P}_{\mathrm{n}}^{(\mathrm{n})}\right\} \triangleleft\left\{\mathrm{Q}_{\mathrm{n}}^{(\mathrm{n})}\right\}$. We still have to show that (3.4) is equivalent to $(3.9)$ and $(3.10)$.

Let $0<\delta<1$. For $\left|\log \mathrm{r}_{\mathrm{ni}}\right| \leq \delta$ we have the expansion

$$
\begin{aligned}
\log r_{n i} & =2 \log \left\{1+\left(q_{n i}^{\frac{1}{2}}-p_{n i}^{\frac{1}{2}}\right) p_{n i}^{-\frac{1}{2}}\right\} \\
& =2\left(q_{n i}^{\frac{1}{2}}-p_{n i}^{\frac{1}{2}}\right) p_{n i}^{-\frac{1}{2}}-\left(q_{n i}^{\frac{1}{2}}-p_{n i}^{\frac{1}{2}}\right)^{2} p_{n i}^{-1}\left(1+p_{n i \delta}\right)
\end{aligned}
$$

with $\left|\rho_{\text {ni } \delta}\right|<2 \delta$. Thus

$$
\begin{aligned}
\int_{\left|\log r_{n i}\right| \leq \delta}\left(\log r_{n i}\right) p_{n i} d \mu_{n i}= & \\
=-2 \int_{\left|\log r_{n i}\right| \leq \delta}\left(q_{n i}^{\frac{1}{2}}-p_{n i}^{\frac{1}{2}}\right)^{2} d \mu_{n i}+ & \int_{\left|\log r_{n i}\right| \leq \delta}\left(q_{n i}-p_{n i}\right) d \mu_{n i}+ \\
& -\int_{\left|\log r_{n i}\right| \leq \delta} \rho_{n i \delta}\left(q_{n i}^{\frac{1}{2}}-p_{n i}^{\frac{1}{2}}\right)^{2} d \mu_{n i} \cdot
\end{aligned}
$$


9

Since by (3.7)

$$
\lim _{n \rightarrow \infty}\left\{\sum_{i=1}^{n} \int_{\left|\log r_{n i}\right| \leq \delta}\left(q_{n i}^{\frac{1}{2}}-p_{n i}^{\frac{1}{2}}\right)^{2} d \mu_{n i}-\sum_{i=1}^{n} H^{2}\left(P_{n i}, Q_{n i}\right)\right\}=0
$$

and by $(3.8),\left\{\mathrm{P}_{\mathrm{n}}^{(\mathrm{n})}\right\} \triangleleft\left\{\mathrm{Q}_{\mathrm{n}}^{(\mathrm{n})}\right\}$ and $(2.1)$

$$
\sum_{i=1}^{n} \int_{\left|\log r_{n i}\right| \leq \delta}\left(q_{n i}-p_{n i}\right) d \mu_{n i}=-\sum_{i=1}^{n} \int_{\left|\log r_{n i}\right|>\delta}\left(q_{n i}-p_{n i}\right) d \mu_{n i} \rightarrow 0
$$

for $\mathrm{n} \rightarrow \infty$, we have

$$
\text { (3.12) } \begin{aligned}
& \lim _{\delta \downarrow 0} \limsup _{n \rightarrow \infty}\left|\sum_{i=1}^{n} \int_{\left|\log r_{n i}\right| \leq \delta}\left(\log r_{n i}\right) d P_{n i}+2 \sum_{i=1}^{n} H^{2}\left(P_{n i}, Q_{n i}\right)\right| \leq \\
& \leq 1 m_{\delta \downarrow 0} \limsup _{n \rightarrow \infty} 2 \delta \sum_{i=1}^{n} H^{2}\left(P_{n i}, Q_{n i}\right)=0,
\end{aligned}
$$

where we have used $(1.6)$. Similarly,

$$
\begin{aligned}
& \lim _{\delta \downarrow 0} \limsup _{\mathrm{n} \rightarrow \infty} \sum_{i=1}^{\mathrm{n}}\left\{\int_{\left|\log \mathrm{r}_{\mathrm{ni}}\right| \leq \delta}\left(\log \mathrm{r}_{\mathrm{ni}}\right) \mathrm{dP} \mathrm{ni}_{\mathrm{n}}\right\}^{2} \leq \\
& \leq \lim _{\delta \downarrow 0} \limsup _{\mathrm{n} \rightarrow \infty} \delta \sum_{\mathrm{i}=1}^{\mathrm{n}}\left|\int_{\left|\log \mathrm{r}_{\mathrm{ni}}\right| \leq \delta}\left(\log \mathrm{r}_{\mathrm{ni}}\right) \mathrm{dP} \mathrm{ni}_{\mathrm{i}}\right| \leq \\
& \leq \lim _{\delta \downarrow 0} \limsup _{\mathrm{n} \rightarrow \infty} \delta(2+2 \delta) \sum_{\mathrm{i}=1}^{\mathrm{n}} \mathrm{H}^{2}\left(\mathrm{P}_{\mathrm{ni}}, \mathrm{Q}_{\mathrm{ni}}\right)=0 .
\end{aligned}
$$

Finally (3.11) implies that for $\left|\log r_{n i}\right| \leq \delta<1$

$$
\left(\log r_{n i}\right)^{2}=4\left(q_{n i}^{\frac{1}{2}}-p_{n i}^{\frac{1}{2}}\right)^{2} p_{n i}^{-1}+\bar{\rho}_{n i \delta}\left(q_{n i}^{\frac{1}{2}}-p_{n i}^{\frac{1}{2}}\right)^{2} p_{n i}^{-1}
$$

with $\left|\bar{\rho}_{\text {ni }}\right|<10 \delta$. Hence, in view of (3.7) and (1.6),

(3.14) $\lim _{\delta \downarrow 0} \limsup _{n \rightarrow \infty}\left|\sum_{i=1}^{n} \int_{\left|\log r_{n i}\right| \leq \delta}\left(\log r_{n i}\right)^{2} d P_{n i}-4 \sum_{i=1}^{n} H^{2}\left(P_{n i}, Q_{n i}\right)\right|=0$. 
The equivalence of (3.4) to (3.9) and (3.10) is now an immediate consequence of $(3.12),(3.13)$ and $(3.14)$. The theorem is proved.

In the one sample case where, for each $n, x_{n 1}, \ldots, x_{n n}$ are identically distributed, condition (3.3) is implied by (3.2) and theorem 2 slightly simplifies. This remains true in the $k$ sample case $(k \geq 2)$ provided all sample sizes tend to infinity.

The first part of the proof of theorem 2 also shows that the conditions (2.3) and (2.5) in corollary 1 may be replaced by the single condition

$$
\lim _{n \rightarrow \infty} \sum_{i=1}^{n} \int_{\left|q_{n i}-p_{n i}\right| \geq c_{n} p_{n i}}\left(q_{n i}^{\frac{1}{2}}-p_{n i}^{\frac{1}{2}}\right)^{2} d \mu_{n i}=0 \text { whenever } c_{n} \rightarrow \infty \text {. }
$$

The proof of theorem 2 could also be given in a more roundabout way. Introducing the $r \cdot v \cdot$ 's

$$
w_{n i}=2\left\{q_{n i}\left(x_{n i}\right) / p_{n i}\left(x_{n i}\right)\right\}^{\frac{1}{2}}-2, \quad i=1, \ldots, n ; n=1,2, \ldots,
$$

one shows that $L\left(\sum_{i=1}^{n} w_{n i} \mid P_{n}^{(n)}\right) \rightarrow_{w} N\left(-\frac{1}{4} \sigma^{2} ; \sigma^{2}\right)$ iff $L\left(\Lambda_{n} \mid P_{n}^{(n)}\right) \rightarrow_{w} N\left(-\frac{1}{2} \sigma^{2} ; \sigma^{2}\right)$, provided the respective $u \cdot a \cdot n$. conditions are satisfied. It is then not difficult to prove that the weak convergence of $\sum_{i=1}^{n} W_{n i}$ and the u.a.n. condition on the summands are equivalent to (3.4) and (3.7). In this proof (3.7) appears as the Lindeberg condition in the central limit theorem applied to $\sum_{i=1}^{\mathrm{n}} \mathrm{W}_{\mathrm{ni}}$.

The equivalence of both weak convergence results has first been proved by LECAM $([3],[4])$. The initial assumptions $\lim _{n \rightarrow \infty} \sup _{1 \leq i \leq n} H^{2}\left(P_{n i}, Q_{n i}\right)=0$ and $1 \limsup _{n \rightarrow \infty}\left\|P_{n}^{(n)}-Q_{n}^{(n)}\right\|<1$ made by LeCam are not restrictive since they are implied by our condition (3.7) and the contiguity of $\left\{P_{n}^{(n)}\right\}$ and $\left\{Q_{n}^{(n)}\right\}$, respectively. One part of this proof is also contained in HÁJEK \& ŠIDÁK [1].

\section{REFERENCES}

[1] HĀJEK, J. \& Z. ŠIDĀK (1967), Theory of rank tests, Academic Press, New York. 
[2] LECAM, L. (1960), Locally asymptotically normal families of distributions, Univ. California Publ. Statist., 3 , 37-98, University of California Press.

[3] LECAM, L. (1966), Likelihood functions for large numbers of independent observations, Research papers in statistics (Festschrift for J. Neyman), 167-187, F.N. David (ed.), Wiley, New York.

[4] LECAM, L. (1969), Théorie asymptotique de la décision statistique, Les Presses de 1 'Université de Montréal.

[5] LOÈVE, M. (1963), Probability theory (3rd ed.), Van Nostrand, New York.

[6] ROUSSAS, G.G. (1972), Contiguity of probability measures: some applications in statistics, Cambridge University Press.

[7] WITTING, H. \& G. NÖLLE (1970), Angewandte mathematische Statistik, Teubner, Stuttgart. 
